122.

\title{
ON THE HOMOGRAPHIC TRANSFORMATION OF A SURFACE OF THE SECOND ORDER INTO ITSELF.
}

[From the Philosophical Magazine, vol. vi. (1853), pp. 326-333.]

THE following theorems in plane geometry, relating to polygons of any number (odd or even) of sides, are well known.

"If there be a polygon of $(m+1)$ sides inscribed in a conic, and $m$ of the sides pass through given points, the $(m+1)$ th side will envelope a conic having double contact with the given conic." And "If there be a polygon of $(m+1)$ sides inscribed in a conic, and $m$ of the sides touch conics having double contact with the given conic, the $(m+1)$ th side will envelope a conic having double contact with the given conic." The second theorem of course includes the first, but I state the two separ 'ely for the sake of comparison with what follows.

As regards the corresponding theory in geometry of three dimensions, Sir W. Hamilton has given a theorem relating to polygons of an odd number of sides, which may be thus stated: "If there be a polygon of $(2 m+1)$ sides inscribed in a surface of the second order, and $2 m$ of the sides pass through given points, the $(2 m+1)$ th side will constantly touch two surfaces of the second order, each of them intersecting the given surface of the second order in the same four lines ${ }^{1}$."

${ }^{1}$ See Phil. Mag. vol. xxxv. [1849] p. 200. The form in which the theorem is exhibited by Sir W. Hamilton is somewhat different; the surface containing the angles is considered as being an ellipsoid, and the two surfaces touched by the last or $(2 m+1)$ th side of the polygon are spoken of as being an ellipsoid, and a hyperboloid of two sheets, having respectively double contact with the given ellipsoid: the contact is, in fact, a quadruple contact at the sam? four points; real as regards two of them in the case of the ellipsoid, and as regards the other two in the case of the hyperboloid of two sheets; and a quadruple contact is the coincidence of four generating lines belonging two and two to the two series of generating lines, these generating lines being of course (in the case considered by Sir W. Hamilton) all of them imaginary.

C. II. 
The entire theory depends upon what may be termed the transformation of a surface of the second order into itself, or analytically, upon the transformation of a quadratic form of four indeterminates into itself. I use for shortness the term transformation simply; but this is to be understood as meaning a homographic transformation, or in analytic language, a transformation by means of linear substitutions. It will be convenient to remark at the outset, that if two points of a surface of the second order have the relation contemplated in the data of Sir W. Hamilton's theorem (viz. if the line joining the two points pass through a fixed point), the transformation is, using the language of the Recherches Arithmétiques, an improper one, but that the relation contemplated in the conclusion of the theorem (viz. that of two points of a surface of the second order, connected by a line touching two surfaces of the second order each of them intersecting the given surface of the second order in the same four lines) depends upon a proper transformation; and that the circumstance that an even number of improper transformations is required in order to make a proper transformation (that this circumstance, I say), is the reason why the theorem applies to polygons in which an even number of sides pass through fixed points, that is, to polygons of an odd number of sides.

Consider, in the first place, two points of a surface of the second order such that the line joining them passes through a given point. Let $x, y, z, w$ be current coordinates $^{1}$, and let the equation of the surface be

$$
(a, \ldots)(x, y, z, w)^{2}=0
$$

and take for the coordinates of the two points on the surface $x_{1}, y_{1}, z_{1}, w_{1}$ and $x_{2}, y_{2}, z_{3}, w_{2}$, and for the coordinates of the fixed point $\alpha, \beta, \gamma, \delta$. Write for shortness

$$
\begin{aligned}
& (a, \ldots)(\alpha, \beta, \gamma, \delta)^{2}=p, \\
& (a, \ldots)(\alpha, \beta, \gamma, \delta)\left(x_{1}, y_{1}, z_{1}, w_{1}\right)=q_{1},
\end{aligned}
$$

then the coordinates $x_{2}, y_{2}, z_{2}, w_{2}$ are determined by the very simple formulæ

$$
\begin{aligned}
& x_{2}=x_{1}-\frac{2 \alpha}{p} q_{1}, \\
& y_{2}=y_{1}-\frac{2 \beta}{p} q_{1}, \\
& z_{2}=z_{1}-\frac{2 \gamma}{p} q_{1}, \\
& w_{2}=w_{1}-\frac{2 \delta}{p} q_{1} .
\end{aligned}
$$

1 Strictly speaking, it is the ratios of these quantities, e.g. $x: w, y: w, z: w$, which are the coordinates, and consequently, even when the point is given, the values $x, y, z, w$ are essentially indeterminate to a factor près. So that in assuming that a point is given, we should write $x: y: z: w=\alpha: \beta: \gamma: \delta$; and that when a point is obtained as the result of an analytical process, the conclusion is necessarily of the form just mentioned: but when this is once understood, the language of the text may be properly employed. It may be proper to explain here a notation made use of in the text: taking for greater simplicity the case of forms of two variables, $(l, m)(x, y)$ means $l x+m y ;(a, b, c)(x, y)^{2}$ means $a x^{2}+2 b x y+c y^{2} ;(a, b, c)(\xi, \eta)(x, y)$ means $a \xi x+b(\xi y+\eta x)+c \eta y$. The system of coefficients may frequently be indicated by a single coefficient only: thus in the text $(a, \ldots)(x, y, z, x)^{2}$ stands for the most general quadratic function of four variables. 
In fact, these values satisfy identically the equations

$$
\left\|\begin{array}{cccc}
x_{2}, & y_{2}, & z_{2}, & w_{2} \\
x_{1}, & y_{1}, & z_{1}, & w_{1} \\
\alpha, & \beta, & \gamma, & \delta
\end{array}\right\|=0
$$

that is, the point $\left(x_{2}, y_{2}, z_{2}, w_{2}\right)$ will be a point in the line joining $\left(x_{1}, y_{1}, z_{1}, w_{1}\right)$ and $(\alpha, \beta, \gamma, \delta)$. Moreover,

$$
\begin{aligned}
(a, \ldots)\left(x_{2}, y_{2}, z_{2}, w_{2}\right)^{2}= & (a, \ldots)\left(x_{1}, y_{1}, z_{1}, w_{1}\right)^{2} \\
& -\frac{4 q_{1}}{p}(a, \ldots)(\alpha, \beta, \gamma, \delta)\left(x_{1}, y_{1}, z_{1}, w_{1}\right) \\
& +\frac{4 q_{1}^{2}}{p^{2}}(a, \ldots)(\alpha, \beta, \gamma, \delta)^{2} \\
= & (a, \ldots)\left(x_{1}, y_{1}, z_{1}, w_{1}\right)^{2}-\frac{4 q_{1}}{p} q_{1}+\frac{4 q_{1}^{2}}{p^{2}} p
\end{aligned}
$$

that is,

$$
(a, \ldots)\left(x_{2}, y_{2}, z_{2}, w_{2}\right)^{2}=\quad(a, \ldots)\left(x_{1}, y_{1}, z_{1}, w_{1}\right)^{2} \text {; }
$$

so that $x_{1}, y_{1}, z_{1}, w_{1}$ being a point on the surface, $x_{2}, y_{2}, z_{2}, w_{2}$ will be so too. The equation just found may be considered as expressing that the linear equations are a transformation of the quadratic form $(a, \ldots)(x, y, z, w)^{2}$ into itself. If in the system of linear equations the coefficients on the right-hand side were arranged square-wise, and the determinant formed by these quantities calculated, it would be found that the value of this determinant is -1 . The transformation is on this account said to be improper. If in a system of linear equations for the transformation of the form into itself the determinant (which is necessarily +1 or else -1 ) be +1 , the transformation is in this case said to be proper.

We have next to investigate the theory of the proper transformations of a quadratic form of four indeterminates into itself. This might be done for the absolutely general form by means of the theory recently established by M. Hermite, but it will be sufficient for the present purpose to consider the system of equations for the transformation of the form $x^{2}+y^{2}+z^{2}+w^{2}$ into itself given by me some years since. (Crelle, vol. XxxII. $[1846]$ p. $119,[52]\left({ }^{1}\right)$.

I proceed to establish (by M. Hermite's method) the formulæ for the particular case in question. The thing required is to find $x_{2}, y_{2}, z_{2}, w_{2}$ linear functions of $x_{1}, y_{1}, z_{1}, w_{1}$, such that

Write

$$
x_{2}{ }^{2}+y_{2}{ }^{2}+z_{2}^{2}+w_{2}^{2}=x_{1}^{2}+y_{1}^{2}+z_{1}^{2}+w_{1}^{2} .
$$

$$
x_{1}+x_{2}=2 \xi, \quad y_{1}+y_{2}=2 \eta, \quad z_{1}+z_{2}=2 \zeta, \quad w_{1}+w_{2}=2 \omega ;
$$

1 It is a singular instance of the way in which different theories connect themselves together, that the formulæ in question were generalizations of Euler's formulæ for the rotation of a solid body, and also are formulæ which reappear in the theory of quaternions; the general formulæ cannot be established by any obvious generalization of the theory of quaternions. 
then putting $x_{2}=2 \xi-x_{1}$, \&c., the proposed equation will be satisfied if only

$$
\xi^{2}+\eta^{2}+\zeta^{2}+\omega^{2}=\xi x_{1}+\eta y_{1}+\zeta z_{1}+\omega w_{1},
$$

which will obviously be the case if

$$
\begin{aligned}
& x_{1}=\xi+\nu \eta-\mu \zeta+a \omega \\
& y_{1}=-\nu \xi+\eta+\lambda \zeta+b \omega \\
& z_{1}=\mu \xi-\lambda \eta+\zeta+c \omega \\
& w_{1}=-a \xi-b \eta-c \zeta+\omega,
\end{aligned}
$$

where $\lambda, \mu, \nu, a, b, c$ are arbitrary.

Write for shortness

$$
a \lambda+b \mu+c \nu=\phi, 1+\lambda^{2}+\mu^{2}+\nu^{2}+a^{2}+b^{2}+c^{2}+\phi^{2}=k,
$$

then we have

$$
\begin{aligned}
& k \xi=\left(1+\lambda^{2}+b^{2}+c^{2}\right) x_{1}+(\lambda \mu-\nu-a b-c \phi) y_{1}+(\nu \lambda+\mu-c a+b \phi) z_{1}+(b \nu-c \mu-a-\lambda \phi) w_{1}, \\
& k \eta=(\lambda \mu+\nu-a b+c \phi) x_{1}+\left(1+\mu^{2}+c^{2}+a^{2}\right) y_{1}+(\mu \nu-\lambda-b c+a \phi) z_{1}+(c \lambda-a \nu-b-\mu \phi) w_{1}, \\
& k \zeta=(\nu \lambda-\mu-c a-b \phi) x_{1}+(\mu \nu+\lambda-b c+a \phi) y_{1}+\left(1+\nu^{2}+a^{2}+b^{2}\right) z_{1}+(a \mu-b \lambda-c-\nu \phi) w_{1}, \\
& k w=(b \nu-c \mu+a+\lambda \phi) x_{1}+(c \lambda-a \nu+b+\mu \phi) y_{1}+(a \mu-b \nu+c+\nu \phi) z_{1}+\left(1+\lambda^{2}+\mu^{2}+\nu^{2}\right) w_{1}
\end{aligned}
$$

and from these we obtain

$$
\begin{aligned}
& k x_{2}=\left(1+\lambda^{2}+b^{2}+c^{2}-\mu^{2}-\nu^{2}-a^{2}-\phi^{2}\right) x_{1}+2(\lambda \mu-\nu-a b-c \phi) y_{1}+2(\nu \lambda+\mu-c a+b \phi) z_{1} \\
&+2(b \nu-c \mu-a-\lambda \phi) w_{1} \\
& k y_{2}=2(\lambda \mu+\nu-a b+c \phi) x_{1}+\left(1+\mu^{2}+c^{2}+a^{2}-\nu^{2}-\lambda^{2}-b^{2}-\phi^{2}\right) y_{1}+2(\mu \nu-\lambda-b c-a \phi) z_{1} \\
&+2(c \lambda-a \nu-b-\mu \phi) w_{1} \\
& k z_{2}=2(\nu \lambda-\mu-c a-b \phi) x_{1}+2(\mu \nu+\lambda-b c+a \phi) y_{1}+\left(1+\nu^{2}+a^{2}+b^{2}-\lambda^{2}-\mu^{2}-c^{2}-\phi^{2}\right) z_{1} \\
&+2(a \mu-b \lambda-c-\nu \phi) w_{1}
\end{aligned}
$$$$
k w_{2}=2(b \nu-c \mu+a+\lambda \phi) x_{1}+2(c \lambda-a \nu+b+\mu \phi) \bar{y}_{1}+2(a \mu-b \nu+c+\nu \phi) z_{1}
$$$$
+\left(1+\lambda^{2}+\mu^{2}+\nu^{2}-a^{2}-b^{2}-c^{2}-\phi^{2}\right) w_{1}
$$

values which satisfy identically $x_{2}{ }^{2}+y_{2}{ }^{2}+z_{2}{ }^{2}+w_{2}{ }^{2}=x_{1}{ }^{2}+y_{1}{ }^{2}+z_{1}{ }^{2}+w_{1}{ }^{2}$.

Dividing the linear equations by $k$, and forming with the coefficients on the righthand side of the equation so obtained a determinant, the value of this determinant is +1 ; the transformation is consequently a proper one. And conversely, what is very important, every proper transformation may be exhibited under the preceding form ${ }^{1}$.

1 The nature of the reasoning by which this is to be established may be seen by considering the analogous relation for two variables. Suppose that $x_{1}, y_{1}$ are linear functions of $x$ and $y$ such that $x_{1}^{2}+y_{1}^{2}=x^{2}+y^{2}$; then if $2 \xi=\dot{x}+x_{1}, 2 \eta=y+y_{1}, \xi$, $\eta$ will be linear functions of $x, y$ such that $\xi^{2}+\eta^{2}=\xi x+\eta y$, or $\xi(\xi-x)+\eta(\eta-y)=0$; $\xi-x$ must be divisible either by $\eta$ or else by $\eta-y$. On the former supposition, calling the quotient $\nu$, we have $x=\xi-\nu \eta$, and thence $y=\nu \xi+\eta$, leading to a transformation such as is considered in the text, and which is a proper transformation; the latter supposition leads to an improper transformation. The given transformation, assumed to be proper, exists and cannot be obtained from the second supposition; it nust therefore be obtainable from the first supposition, i.e. it is a transformation which may be exhibited under a form such as is considered in the text. 
Next considering the equations connecting $x, y, z, w$ with $\xi, \eta, \zeta, \omega$, we see that

$$
\begin{aligned}
x_{1}{ }^{2}+y_{1}{ }^{2}+z_{1}{ }^{2}+w_{1}{ }^{2} & =(\quad \xi+\nu \eta-\mu \zeta+a \omega)^{2} \\
& +(-\nu \xi+\eta+\lambda \zeta+b \omega)^{2} \\
& +(\mu \xi-\lambda \eta+\zeta+c \omega)^{2} \\
& +(-a \xi-b \eta-c \zeta+\omega)^{2} .
\end{aligned}
$$

We are thus led to the discussion (in connexion with the question of the transformation into itself of the form $x^{2}+y^{2}+z^{2}+w^{2}$ ) of the new form

$$
\begin{array}{r}
\quad(\quad x+\nu y-\mu z+a w)^{2} \\
+(-\nu x+y+\lambda z+b w)^{2} \\
+(\mu x-\lambda y+z+c w)^{2} \\
+(-a x-b y-c z+w)^{2}
\end{array}
$$

or, as it may also be written,

$$
\left(x^{2}+y^{2}+z^{2}+w^{2}\right)+(\nu y-\mu z+a w)^{2}+(\lambda z-\nu x+b w)^{2}+(\mu x-\lambda y+c w)^{2}+(a x+b y+c z)^{2} .
$$

Represent for a moment the forms in question by $U, V$, and consider the surfaces $U=0, V=0$. If we form from this the surface $V+q U=0$, and consider the discriminant of the function on the left-hand side, then putting for shortness

$$
\kappa=\lambda^{2}+\mu^{2}+\nu^{2}+a^{2}+b^{2}+c^{2},
$$

this discriminant is

$$
\left\{(q+1)^{2}+\kappa(q+1)+\phi^{2}\right\}^{2},
$$

which shows that the surfaces intersect in four lines. Suppose the discriminant vanishes; we have for the determination of $q$ a quadratic equation, which may be written

$$
q^{2}+(2+\kappa) q+K=0
$$

let the roots of this equation be $q_{1}, q_{11}$; then each of the functions $q_{1} U+V, q_{11} U+V$ will break up into linear factors, and we may write

$$
\begin{aligned}
& q_{1} U+V=R, S_{\prime}, \\
& q_{\prime \prime} U+V=R_{\prime \prime} S_{\prime \prime} .
\end{aligned}
$$

( $U$ and $V$ are of course linear functions of $R, S_{,}$and $R_{\| \prime} S_{\| \prime}$ ) forms which put in evidence the fact of the two surfaces intersecting in four lines.

The equations

$$
x_{1}+x_{2}=2 \xi, \quad y_{1}+y_{2}=2 \eta, \quad z_{1}+z_{2}=2 \zeta, \quad w_{1}+w_{2}=2 \omega,
$$


show that the point $(\xi, \eta, \zeta, \omega)$ lies in the line joining the points $\left(x_{1}, y_{1}, z_{1}, w_{1}\right)$ and $\left(x_{2}, y_{2}, z_{2}, w_{2}\right)$; and to show that this line touches the surface $V=0$, it is only necessary to form the equation of the tangent plane at. the point $(\xi, \eta, \zeta, \omega)$ of the surface in question; this is

$$
(x+\nu y-\mu z+a w)(\xi+\nu \eta-\mu \zeta+a \omega)+\ldots=0 ;
$$

or what is the same thing,

$$
(x+\nu y-\mu z+a w) x_{1}+\ldots=0,
$$

which is satisfied by writing $\left(x_{1}, y_{1}, z_{1}, w_{1}\right)$ for $(x, y, z, w)$, that is, the tangent plane of the surface contains the point $\left(x_{1}, y_{1}, z_{1}, w_{1}\right)$. We see, therefore, that the line through $\left(x_{1}, y_{1}, z_{1}, w_{1}\right)$ and $\left(x_{2}, y_{2}, z_{2}, w_{2}\right)$ touches the surface $V=0$ at the point $(\xi, \eta, \zeta, \omega)$.

Write now

$$
a^{\prime}=\frac{-\lambda}{\phi}, \quad b^{\prime}=\frac{-\mu}{\phi}, \quad c^{\prime}=\frac{-\nu}{\phi}, \quad \lambda^{\prime}=\frac{-a}{\phi}, \quad \mu^{\prime}=\frac{-b}{\phi}, \quad \nu^{\prime}=\frac{-c}{\phi} ;
$$

if we derive from the coordinates $x_{1}, y_{1}, z_{1}, w_{1}$, by means of these coefficients $a^{\prime}, b^{\prime}, c^{\prime}, \lambda^{\prime}, \mu^{\prime}, \nu^{\prime}$, new coordinates in the same way as $x_{2}, y_{2}, z_{2}, w_{2}$ were derived by means of the coefficients $a, b, c, \lambda, \mu, \nu$, the coordinates so obtained are $-x_{2},-y_{2},-z_{2},-w_{2}$, i.e. we obtain the very same point $\left(x_{2}, y_{2}, z_{2}, w_{2}\right)$ by means of the coefficients $(a, b, c, \lambda, \mu, \nu)$, and by means of the coefficients $\left(a^{\prime}, b^{\prime}, c^{\prime}, \lambda^{\prime}, \mu^{\prime}, \nu^{\prime}\right)$. Call $\xi^{\prime}, \eta^{\prime}, \xi^{\prime}, \omega^{\prime}$ what $\xi, \eta, \xi, \omega$ become when the second system of coefficients is substituted for the first; the point $\xi^{\prime}, \eta^{\prime}, \zeta^{\prime}, \omega^{\prime}$ will be a point on the surface $V^{\prime}=0$, where

$$
\begin{aligned}
V^{\prime}=\phi^{2}\left(x^{2}+y^{2}\right. & \left.+z^{2}+w^{2}\right) \\
& +(-c y+b z-\lambda w)^{2}+(-a z+c x-\mu w)^{2}+(-b x+a y-\nu w)^{2}+(-\lambda x-\mu y-\nu z)^{2}
\end{aligned}
$$

and since

$$
V+V^{\prime}=\kappa\left(x^{2}+y^{2}+z^{2}+w^{2}\right)
$$

and $V=0$ intersects the surface $x^{2}+y^{2}+z^{2}+w^{2}=0$ in four lines, the surface $V^{\prime}=0$ will also intersect this surface in the same four lines. And it is, moreover, clear that the line joining the points $\left(x_{1}, y_{1}, z_{1}, w_{1}\right)$ and $\left(x_{2}, y_{2}, z_{2}, w_{2}\right)$ touches the surface $V^{\prime}=0$ in the point $\left(\xi^{\prime}, \eta^{\prime}, \zeta^{\prime}, \omega^{\prime}\right)$. We thus arrive at the theorem, that when two points of a surface of the second order are so connected that the coordinates of the one point are linear functions of the coordinates of the other point, and the transformation is a proper one, the line joining the two points touches two surfaces of the second order, each of them intersecting the given surface of the second order in the same four lines. Any two points so connected may be said to be corresponding points, or simply a pair. Suppose the four lines and also a single pair is given, it is not for the determination of the other pairs necessary to resort to the two auxiliary surfaces of the second order; it is only necessary to consider each point of the surface as determined by the two generating lines which pass through it; then considering first 
one point of the given pair, and the point the corresponding point to which has to be determined, take through each of these points a generating line, and take also two generating lines out of the given system of four lines, the four generating lines in question being all of them of the same set, these four generating lines intersecting either of the other two generating lines of the given system of four lines in four points. Imagine the same thing done with the other point of the given pair and the required point, we should have another system of four points (two of them of course identical with two of the points of the first-mentioned system of four points); these two systems must have their anharmonic ratios the same, a condition which enables the determination of the generating line in question through the required point: the other generating line through the required point is of course determined in the same manner, and thus the required point (i.e. the point corresponding to any point of the surface taken at pleasure) is determined by means of the two generating lines through such required point.

It is of course to be understood that the points of each pair belong to two distinct systems, and that the point belonging to the one system is not to be confounded or interchanged with the point belonging to the other system. Consider, now, a point of the surface, and the line joining such point with its corresponding point, but let the corresponding point itself be altogether dropped out of view. There are two directions in which we may pass along the surface to a consecutive point, in such manner that the line belonging to the point in question may be intersected by the line belonging to the consecutive point. We have thus upon the surface two series of curves, such that a curve of each series passes through a point chosen at pleasure on the surface. The lines belonging to the curves of the one series generate a series of developables, the edges of regression of which lie on one of the surfaces intersecting the surface of the second order in the four given lines; the lines belonging to the curves of the other series generate a series of developables, the edges of regression of which lie on the other of the surfaces intersecting the surface in the four given lines; the general nature of the system may be understood by considering the system of normals of a surface of the second order. Consider, now, the surface of the second order as given, and also the two surfaces of the second order intersecting it in the same four lines; from any point of the surface we may draw to the auxiliary surfaces four different tangents; but selecting any one of these, and considering the other point in which it intersects the surface as the point corresponding to the first-mentioned point, we may, as above, construct the entire system of corresponding points, and then the line joining any two corresponding points will be a tangent to the two auxiliary surfaces; the system of tangents so obtained may be called a system of congruent tangents. Now if we take upon the surface three points such that the first and second are corresponding points, and that the second and third are corresponding points, then it is obvious that the third and first are corresponding points;-observe that the two auxiliary surfaces for expressing the correspondence between the first and second point, those for the second and third point, and those for the third and first point, meet the surface, the two auxiliary surfaces of each pair in the same four lines, but that these systems of four lines are different 
for the different pairs of auxiliary surfaces. The same thing of course applies to any number of corresponding points. We have thus, finally, the theorem, if there be a polygon of $(m+1)$ sides inscribed in a surface of the second order, and the first side of the polygon constantly touches two surfaces of the second order, each of them intersecting the surface of the second order in the same four lines (and the side belong always to the same system of congruent tangents), and if the same property exists with respect to the second, third, \&c.... and $m$ th side of the polygon, then will the same property exist with respect to the $(m+1)$ th side of the polygon.

We may add, that, instead of satisfying the conditions of the theorem, any two consecutive sides of the polygon, or the sides forming any number of pairs of consecutive sides, may pass each through a fixed point. This is of course only a particular case of the improper transformation of a surface of a second order into itself, a question which is not discussed in the present paper. 